\title{
HYPOPHOSPHATEMIC ONCOGENIC OSTEOMALACIA: CASE REPORT
}

Rafaela Zarpelon Kunz 1,", Tatiana Freitas Tourinho¹, Maria Lucia Lemos Lopes', Maria Odete Esteves Hilario¹, Eduardo Rosa de Oliveira ${ }^{1}$, Thiago Willers ${ }^{1}$, Rafael Coradin ${ }^{1}$, Gilberto Scanagatta ${ }^{1}$, Raissa Velasques de Figueiredo ${ }^{1}$, Gabriela Sasso Padilha ${ }^{1}$, Bruno Trevisann ${ }^{1}$ Luana Ribeiro Carlos ${ }^{1}$

1. Universidade Federal de Ciências da Saúde de Porto Alegre, Porto Alegre (RS), Brazil.

*Corresponding author: consultoriowillers@gmail.com

\section{BACKGROUND}

Hypophosphatemic oncogenic osteomalacia ( $\mathrm{HOO})$ is a tumor-induced, soft-tissue or bone-induced paraneoplastic syndrome. It presents with pain and fractures, accompanied by hypophosphatemia and plasma concentrations of $1.25(\mathrm{OH}) 2 \mathrm{D} 3$ inappropriately normal or decreased. This report presents the case of a patient with $\mathrm{HOO}$, with a marked degree of osteoporosis, and who showed improvement in bone densitometry parameters after tumor removal in the inguinal region and vitamin $\mathrm{D}$ replacement.

\section{CASE REPORT}

Male patient, 45 years old, previously healthy. Currently using alendronate $70 \mathrm{mg} /$ week, vitamin D321.000 IU/week and calcium carbonate 500 mg twice a day. Since 2010, he had hypovitaminosis D, hypophosphatemia and hypophosphaturia, with clinical and radiological signs suggestive of osteomalacia. Upon examination, he presented thoracic deformities (pectus carinatum), marked thoracic kyphosis and rectification of the cervical and lumbar spine. In addition, height reduction from 144 to $137 \mathrm{~cm}$ in 4 years. Initial densitometry with T score L1-L4 of -2.5, femoral neck -3.3 and total femur -3.6. During investigation exams, a hypermetabolic nodule was found in the left inguinal region near the base of the scrotum of the midline and a metabolically active lymph node in the right inguinal region. Inguinal tumor excision was performed in August 2015, with a pathological examination showing a phosphaturic mesenchymal tumor. Hyperparathyroidism discarded due to laboratory tests showing normal parathyroid hormone (PTH) and parathyroid scintigraphy without signs suggestive of hyperfunctioning parathyroid tissue. Final diagnosis suggestive of hypophosphatemic oncogenic osteomalacia. After removal of the tumor and beginning of vitamin D replacement, the patient progressed with significant improvement in metabolic laboratory tests and osteoporosis. Current densitometry with measurements of $\mathrm{T}$ score of neck of femur of -1.7 , total femur of -0.8 and forearm of -0.7 .

\section{CONCLUSION}

Hypophosphatemic oncogenic osteomalacia is a subtype of acquired osteomalacia, characterized as a paraneoplastic phenomenon, in which musculoskeletal disease has greater clinical significance than the tumor itself. Its greatest feature is the complete reversal of the metabolic and musculoskeletal condition with the removal of the tumor. 